\title{
Evaluation of the Overweight/Obese Child - Practical Tips for the Primary Health Care Provider: Recommendations from the Childhood Obesity Task Force of the European Association for the Study of Obesity
}

\author{
Jennifer L. Baker ${ }^{a} \quad$ Nathalie J. Farpour-Lambert ${ }^{b}$ Paulina Nowicka ${ }^{c}$ \\ Angelo Pietrobellid ${ }^{d}$ Ram Weiss ${ }^{\mathrm{e}}$ \\ ${ }^{a}$ Institute of Preventive Medicine, Centre for Health and Society, Copenhagen, Denmark \\ ${ }^{b}$ Obesity Care Program, Pediatric Cardiology Unit, Department of Child and Adolescent, University Hospital of Geneva, Switzerland \\ ${ }^{c}$ Childhood Obesity Unit, University Hospital, Malmö, Sweden \\ ${ }^{\mathrm{d}}$ Pediatric Unit, Verona University Medical School, Italy \\ ${ }^{\text {e }}$ Department of Human Metabolism and Nutrition, Hebrew University School of Medicine, Jerusalem, Israel
}

Key Words

Childhood obesity · Children · Guidelines ·

Obesity management

\section{Summary}

The prevalence of obesity among children and adolescents is on the rise. The majority of overweight or obese children are treated by primary health care providers including paediatricians, family practitioners, dieticians, nurses, and school health services - and not by specialists. The majority of obese children have no underlying medical disorder causing their obesity yet a significant proportion might suffer from obesity-related co-morbidities. This text is aimed at providing simple and practical tools for the identification and management of children with or at risk of overweight and obesity in the primary care setting. The tips and tools provided are based on data from the recent body of work that has been published in this field, official statements of several scientific societies along with expert opinion provided by the members of the Childhood Obesity Task Force (COTF) of the European Association for the Study of Obesity (EASO). We have attempted to use an evidence-based approach while allowing flexibility for the practicing clinician in domains where evidence is currently lacking and ensuring that treating the obese child involves the entire family as well.

\section{Introduction}

The majority of overweight or obese children are treated by primary health care providers including paediatricians, family practitioners, dieticians, nurses, and school health services and not by specialists. Patient encounters in the primary care setting are typically limited in time and are in the context of an acute illness. Given these constraints, it is challenging to identify and treat children suffering from excess weight. Oftentimes the patient and family are unaware of the problem, which makes raising the issue of weight problematic for the practitioner. For these reasons, simple and practical tools for the identification and management of children with or at risk of overweight and obesity are needed in the primary care setting.

Practitioners need these tools because it is critical to treat these children early in life. Excess weight negatively affects the child's current physical and psychosocial health [1] as well as compromises their future health [2]. Unlike many paediatric health problems, childhood obesity has multiple causes and requires a broad treatment approach encompassing the patient, the family, and the environment. Additionally, the majority of its complications are asymptomatic. Despite these challenges, a motivational approach along with simple lifestyle changes, in particular if introduced early, can significantly benefit the health and well-being of the overweight child and may potentially benefit other family members.

In recent years, numerous guidelines have been published for the care of the obese child [3-6]. The aim of this document, however, is to provide a simple and practical approach for the assessment and management of the overweight or

\begin{tabular}{ll}
\hline KARGER & $\oplus$ c 2010 S. Karger GmbH, Freiburg \\
Fax +497614520714 & Accessible online at: \\
$\begin{array}{l}\text { Information@Karger.de } \\
\text { www.karger.com }\end{array}$ & www.karger.com/ofa
\end{tabular}




\section{ASSESSMENT OF THE OVERWEIGHT CHILD}

History Main Points

- Pregnancy history - maternal gestational diabetes (GDM), impaired intra uterine growth (IUGR), birth weight (focus on identifying infants born small for gestational age $(S G A))$.

- Assess and plot previous anthropometric data

- Parental and sibling anthropometrics

- Parental and sibling relevant health conditions

- Use of medications by the child

- Review of systems - sleep patterns, mood assessment, physical limitations

Physical examination main points

- Weigh and assess height, calculate body mass index (BMI) and plot them !

- Assess waist circumference

- Search for dysmorphism or stigmata of underlying medical conditions

- Measure blood pressure correctly

- Look for acanthosis nigricans and for signs of hyperandrogenism in girls

Nutritional assessment main tips.

- Establish a steady meal schedule

- Promote eating in the kitchen with the family

- Avoid sweetened beverages! Recommend drinking water.

- Promote consumption of healthy foods - fruit and vegetables, grains

- Promote consumption of complex carbohydrates instead of simple ones

Physical activity main tips

- Promote walking or biking to school

- Promote participation in any moderate to vigorous activity, even in small amounts

- Reduce TV/computer time to $<2$ hours per day

- Suggest activities with parents or friends

- Recommended activity is at least 1 hour per day, yet any activity counts and helps.

Do not forget!

- Set realistic goals and expectations of the child and family

- Emphasize the parental role in making changes and providing a role model

- Emphasize that weight management is a lifelong process and not a brief period of modifications

- Provide positive feedbacks for changes made while keeping to repeat the main messages

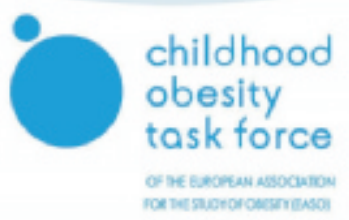

Fig. 1. Checklist of the Childhood Obesity Task Force Guidelines. 
obese child tailored specifically for the primary care setting. Importantly, this approach is also relevant for siblings of the obese child and may help prevent obesity in at-risk patients. The approach presented here focuses on four main areas: history taking, physical examination, and nutritional and physical activity assessments. A descriptive section is provided for each topic along with key action points and tips which have been developed by experts to maximise benefit given time constraints in the primary care setting. Additionally, a checklist summarising these elements is provided (fig. 1).

\section{Triage of the Child in Relation to Obesity Risk}

At the first visit, the goal of the detailed history and physical examination is to categorise the child to one of three categories:

1. Those who require referral to a specialist - including children with obesity suspected to be associated with a syndrome or an organic disorder. A very small minority of children and adolescents $(<2 \%)$ will be included in this category.

2. Those with suspected complications of overweight or obesity - to be evaluated using additional laboratory and other tests. If such complications are present, referral to a specialist should be strongly considered.

3. Children with the typical mixture of a predisposing genetic background and the impact of the obesogenic environment resulting in obesity. They comprise the vast majority of children who can potentially benefit from repeated brief and focused tips to address their weight problem without further referral.

\section{History Taking in the Obese Child}

A focused and problem-oriented history assessment in the obese child has three major aims: i) to rule out an underlying organic disorder, ii) to identify the presence of co-morbidities, and iii) to assess the risk of developing co-morbidities.

Although only a small minority of children have an underlying disorder as the primary cause of their excess weight, this possibility must not be overlooked because of its serious health consequences. A larger number of children will have obesity-related co-morbidities, and these must be treated as their health consequences are also serious. These two groups of children warrant referral to a paediatric obesity specialist and should not be dealt with in the primary care setting.

In the vast majority of children, the cause of overweight or obesity is a predisposing genetic background (obese parents or other family members) and the impact of the obesogenic environment, leading to sedentary behaviour and increased consumption of calorie-dense food. In these children without manifest problems, the identification of risk factors should raise the index of suspicion for the future development of metabolic complications. Treatment of these children is most successful if motivational techniques are used to encourage parents to adopt lifestyle modifications and familial preventive measures. Principles of motivational approaches and patientcentred communication are beyond the scope of this paper, yet they are critical for the successful adoption of meaningful changes [5]. For further information on how to raise the subject of obesity without offending the child or family and how to motivate them positively, please see table 4 from Barlow et al. [5].

In order to help in the evaluation of the child, a series of questions are provided. Questions 1-4 focus on pregnancy, growth, and family history, which are topics known to have associations with childhood obesity. These questions aim at identifying the 'vulnerability' of the child for developing obesity and its complications. As interventions against obesity in childhood aim at improving fitness as well as weight reduction, a child with a positive history for co-morbidities of insulin resistance may for instance benefit most from such measures. Questions 5 and 6 focus on identifying the presence of obesity-related complications in the child. Answers from these questions allow the practitioner to identify children who may need further evaluation by a specialist or who require further laboratory work-up.

\section{Specific Points}

1. Pregnancy history - ask if there were maternal gestational diabetes or other complications of pregnancy (hypertension, impaired intrauterine growth, birth weight (with focus on identifying those born small or large for gestational age)).

2. Assess and plot previous anthropometric data on appropriate growth charts. Focus on 'catch-up growth' of smaller babies, very early weight gain, and weight accrual prior to pubertal development.

3. Grand-parental, parental, and sibling anthropometrics obesity tends to run in families.

4. Grand-parental, parental and sibling health conditions focus on morbidity related to increased cardiovascular risk such as diabetes, dyslipidaemia, hypertension, positive history for overt cardiovascular disease below 55 years in men and below 65 years in women.

5. Use of medications by the child - specifically chronic use of glucocorticoids and/or psychotropic medications, the use of which is typically related to weight gain.

6. Review of systems - sleep patterns (snoring, nocturnal binge eating, nocturia, duration), physical limitations due to orthopaedic problems, mood assessment, eating patterns (focus in history of dieting and on binging). In female adolescents, the regularity of periods and the presence of hyperandrogenism. 
Table 1. Waist circumference percentiles for European-American children and adolescents, according to sex [adapted from 7] ${ }^{\mathrm{a}}$

\begin{tabular}{llllllll}
\hline \multirow{2}{*}{ Age, years } & \multicolumn{3}{c}{ Percentiles for boys, cm } & & \multicolumn{3}{c}{ Percentiles for girls, cm } \\
\cline { 2 - 3 } \cline { 7 - 8 } & $10^{\text {th }}$ & $50^{\text {th }}$ & $90^{\text {th }}$ & & $10^{\text {th }}$ & $50^{\text {th }}$ & $90^{\text {th }}$ \\
\hline 2 & 42.9 & 47.1 & 50.6 & & 43.1 & 47.4 & 52.5 \\
3 & 44.7 & 49.2 & 54.0 & & 44.7 & 49.3 & 55.4 \\
4 & 46.5 & 51.3 & 57.4 & & 46.3 & 51.2 & 58.2 \\
5 & 48.3 & 53.3 & 60.8 & & 47.9 & 53.1 & 61.1 \\
6 & 50.1 & 55.4 & 64.2 & & 49.5 & 55.0 & 64.0 \\
7 & 51.9 & 57.5 & 67.6 & & 51.1 & 56.9 & 66.8 \\
8 & 53.7 & 59.6 & 71.0 & & 52.7 & 58.8 & 69.7 \\
9 & 55.5 & 61.7 & 74.3 & & 54.3 & 60.7 & 72.6 \\
10 & 57.3 & 63.7 & 77.7 & & 55.9 & 62.5 & 75.5 \\
11 & 59.1 & 65.8 & 81.1 & & 57.5 & 64.4 & 78.3 \\
12 & 60.9 & 67.9 & 84.5 & & 59.1 & 66.3 & 81.2 \\
13 & 62.7 & 70.0 & 87.9 & & 60.7 & 68.2 & 84.1 \\
14 & 64.5 & 72.1 & 91.3 & & 62.3 & 70.1 & 86.9 \\
15 & 66.3 & 74.1 & 94.7 & & 63.9 & 72.0 & 89.8 \\
16 & 68.1 & 76.2 & 98.1 & & 65.5 & 73.9 & 92.7 \\
17 & 69.9 & 78.3 & 101.5 & & 67.1 & 75.8 & 95.5 \\
18 & 71.7 & 80.4 & 104.9 & 68.7 & 77.7 & 98.4 \\
\hline
\end{tabular}

${ }^{a}$ Having a waist circumference greater than the 90 th percentile increases the risk for the presence of cardio-vascular risk factors and insulin resistance.

\section{Physical Examination of the Obese Child}

An obese child or adolescent should undergo a standard physical examination just like any other patient, yet certain elements are specifically relevant for them. In particular, these relate to body composition and fat distribution (anthropometric measurements) as well as ruling out the presence of established obesity-related complications. The search for specific signs of an organic cause of the obesity or its co-morbidities is a major part of the overall risk stratification of the child. It is important to emphasise that all anthropometric and vital sign assessments should be compared to appropriate age and gender references for interpretation.

\section{Specific Points}

1. All paediatric patients should be weighed and have their height measured at least once a year. Calculate the body mass index $\left(\mathrm{BMI}=\right.$ weight in kilograms $/$ height in metres $\left.{ }^{2}\right)$ and plot it on appropriate local BMI centile charts. It is recommended that waist circumference is measured and plotted with appropriate centiles as well (table 1) [5, 7]. The child should stand erect with the abdomen relaxed, arms at the sides, and the feet together. Take the measurement at the end of normal expiration at the narrowest part of the torso at the level of the natural waist between the ribs and the iliac crest [8].
Table 2. Simplified blood pressure references for children and adolescents [adapted from 9] $]^{\mathrm{a}}$

\begin{tabular}{llllll}
\hline \multirow{2}{*}{ Age, years } & \multicolumn{2}{l}{$\begin{array}{l}\text { Values for boys, } \\
\mathrm{mm} \mathrm{Hg}\end{array}$} & & \multicolumn{2}{l}{$\begin{array}{l}\text { Values for girls, } \\
\mathrm{mm} \mathrm{Hg}\end{array}$} \\
\cline { 2 - 3 } \cline { 5 - 6 } \cline { 5 - 6 } & systolic & diastolic & & systolic & diastolic \\
\hline 3 & 100 & 59 & 100 & 61 \\
4 & 102 & 62 & 101 & 64 \\
5 & 104 & 65 & 103 & 66 \\
6 & 105 & 68 & 104 & 68 \\
7 & 106 & 70 & 105 & 69 \\
8 & 107 & 71 & 108 & 71 \\
9 & 109 & 72 & 110 & 72 \\
10 & 111 & 73 & & 112 & 73 \\
11 & 113 & 74 & 114 & 74 \\
12 & 115 & 74 & 116 & 75 \\
13 & 117 & 75 & 117 & 76 \\
14 & 120 & 75 & 119 & 77 \\
15 & 120 & 76 & 120 & 78 \\
16 & 120 & 78 & 120 & 78 \\
17 & 120 & 80 & 120 & 78 \\
18 & 120 & 80 & 120 & 80 \\
\hline
\end{tabular}

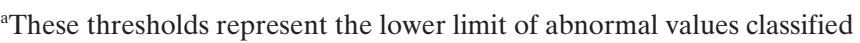
by age and sex. Measurements above these thresholds require an evaluation.
2. Search for stigmata of syndromes associated with obesity developmental delay, dysmorphism, hypogonadism, and purple abdominal striae. Search for the presence of signs associated with simple obesity such as acanthosis nigricans or manifestations of hyperandrogenism in females.

3. Measure blood pressure in a relaxed environment after 10 min of rest and compare it to appropriate references (table 2) [9]. Make sure that the cuff is appropriate for the size of the child.

4. Examine the musculoskeletal system focusing on skeletal deformities and limitation of motion.

\section{Additional Laboratory Work-up}

A basic laboratory evaluation should be performed in obese children (BMI $>97 \%$ for age and gender on local growth charts) to rule out the presence of obesity-related metabolic abnormalities. Abnormalities detected during the laboratory work-up, such as glucose intolerance, dyslipidaemia, or altered liver function, warrant referral to a specialist.

\section{Specific Points}

1. Complete biochemistry including liver function and a fasting lipid profile. Obesity is commonly associated with nonalcoholic fatty liver disease, elevated alanine transaminase (ALT) levels as well as other liver function abnormalities. The typical dyslipidaemia seen in obese children includes 
elevated triglycerides and reduced HDL(high-density lipoprotein)-cholesterol. The presence of this lipid profile is highly suggestive of significant insulin resistance [10].

2. Complete blood count - surprisingly, nutritional deficiencies, and specifically iron deficiency, are common in obese children.

3. Evaluation of glucose metabolism - the sensitivity of a fasting glucose measurement to detect glucose intolerance in overweight/obese children is low. In a child at risk for altered glucose metabolism (severe obesity, positive family history of T2DM or gestational diabetes, presence of acanthosis nigricans, or a suggestive history of polydipsia/polyuria) - a standard oral glucose tolerance test should be performed.

4. Additional tests based on clinical suspicion of a medical disorder/obesity syndrome - such as thyroid function tests, urinary free cortisol collection etc. should be performed as necessary.

At this point the practitioner can establish whether the child should be referred to a specialist (suspected syndromes associated with obesity or the presence of co-morbidity associated with simple obesity) or can be treated locally. Co-morbidities of obesity may be present in a significant number of obese adolescents (impaired glucose tolerance in 10-25\%, hypertension in up to $30 \%$, and non-alcoholic fatty liver disease in up to $8 \%$, depending on the population studied). If the child can be treated locally, then the nutritional and physical activity assessments should be conducted. There is no established 'standard of care' that dictates the frequency of seeing obese children in clinic, yet repetition of nutritional and lifestyle recommendations along with providing positive feedback where appropriate should be performed at least every 4-6 months.

\section{Nutritional Assessment}

The goals of proper nutrition in childhood are to achieve a normal body weight and develop healthy eating habits that will last a lifetime. The first step in successful nutritional counselling is the assessment of calorie intake and food habits. Nutritional assessments should include an evaluation of current intake (most importantly fruit and vegetables, sweetened beverages, fast food, portion sizes) as well as meal and snack patterns (e.g. frequency and quality of meals such as breakfast). Ideally, a clinician with specialised nutrition training, who has the primary responsibility for evaluating and making recommendations related to food behaviour, should perform the assessment.

A number of simple questions asked by any practitioner, however, are adequate for an initial overview of nutritional habits as a comprehensive dietary assessment is difficult to perform and is time-consuming in a primary care setting. The following questions focus on dietary behaviours that have the strongest evidence for associations with energy balance and that are modifiable.

\section{Specific Points}

1. Identify timing and locations of meals - ask about snacking patterns in between meals, during the evening and night. Ask about where the meals are eaten, such as in the kitchen/dining room, living room, or bedroom, and if they are eaten in front of the television or computer.

2. Quantify sweetened beverage consumption - including all soft drinks, fruit juices as well as chocolate milk.

3. Assess positive components of the diet, such as fruit and vegetables, fish, and grains.

4. Assess portion sizes - although the quality of food is critical, quantity can sometimes be an issue as well. Compare portion sizes with parents and other family members.

5. Ask what the child eats during school time.

\section{Practical Tips}

1. Attempt to establish a steady schedule of meals with minimal snacking in between. If snacking occurs, recommend fruit and vegetables.

2. Eat meals in the kitchen/dining room and never in front of the television or computer. Family dinners are strongly recommended.

3. Avoid all sweetened beverages. Water is best for children.

4. Promote consumption of fruit and vegetables as much as possible.

5. Promote consumption of complex carbohydrates instead of simple carbohydrates if possible (e.g. whole wheat products).

6. Encourage consumption of low fat such as lean meat and non-fried foods.

\section{Physical Activity Assessment}

Energy expenditure through physical activity is an important part of the energy balance equation that determines body weight. Fitness, independent of body weight, at any age is associated with a reduced cardiovascular and metabolic risk. Regular activity may also improve the child's overall self-esteem and social interactions. As western lifestyles and modernisation have significantly reduced children's regular daily physical activity levels, the questions focus on everyday activities within the household and at school. These questions about the child's activity allow for identification of areas in which small changes, individually or together with the family or friends, can provide a significant benefit.

\section{Specific Points}

1. Assess hours per day of sedentary activities - such as television watching and computer time. 
2. Ask about any additional activity and participation in organised sports outside of the school system.

3. Ask how the child gets to school - walking, cycling, car, public transport.

4. Assess family activities - do the parents and siblings engage in any physical activity?

\section{Practical Tips}

1. Increase physical activity and fitness - promote walking or cycling to school (assuming this can be performed safely), participating in organised sports activities or even activities like walking a dog. Promote even small amounts of moderate to vigourous activities as much as possible.

2. Reduce sedentary activities (television, computer) - although this is not simple to achieve, a maximum of $2 \mathrm{~h} /$ day of 'media time' should be recommended.

3. Suggest activities that involve parents or friends - like walking or playing games together.

4. The recommended level of activity is a minimum of $1 \mathrm{~h} /$ day every day.

5. Any activity counts! Promote enjoyable and fun activities. Using answers from the nutrition and physical activity assessments, the practitioner can identify the domains in which modifications are most likely to succeed for each child. Tips relevant to these domains can be repeatedly emphasised at each visit. It is important to emphasise to the child and the family that the management of overweight and obesity in children is a long-term process and that immediate drastic success should not be expected. The child and family should be encouraged to maintain small changes for long periods, as these seemingly small modifications can be highly beneficial in the long run.

\section{Goals and Expectations}

Setting realistic goals and expectations can help avoid unnecessary frustrations along the road. For growing children who are overweight or mildly obese, weight maintenance (and not weight loss) is sufficient to improve well-being and the metabolic profile. Acute weight reduction, whether induced by lifestyle modifications or pharmacotherapy, has a very poor long-term success rate and is usually followed by weight regain. Therefore, overweight and obese children and adolescents should be encouraged to make long-lasting lifestyle changes as detailed above. They should be counselled to expect a small, yet steady, weight loss from these types of changes. In adolescents, a weight loss of $1-2 \mathrm{~kg}$ per month is achievable for a limited time in some and may be sustainable. Changes in body composition (such as increasing fat-free mass in exchange for fat or reduction of waist circumference) without weight loss can positively affect well-being and the metabolic profile.

\section{Parents and Family}

The practitioner should repeatedly emphasise the parental role in the process. It cannot be overemphasised at this point that the vast majority of the nutritional and physical activity tips provided to the parents are aimed at the entire family and not solely at the child. Parents are responsible for grocery shopping and meal preparation. They need to be reminded that they serve as role models for their children. Parents should support the child and provide positive feedback. The practitioner should specifically encourage these lifestyle changes in families with children at risk for obesity (overweight or those with obese parents) as prevention of obesity is much easier than reverting it. The potential benefits of these changes for the entire household should be explained and reemphasised repeatedly.

\section{Conclusions}

The main objective of the treatment in overweight and obese children and adolescents is to help them attain and maintain a body weight that will improve their well-being and prevent the development of complications. As the risk of an obese child becoming an obese adult increases from $25 \%$ before 6 years of age to $75 \%$ during adolescence, the treatment must be initiated as early as possible. This is of particular importance as co-morbidities may appear during childhood even before the child is classified as being obese. The treatment described here aims at changing behaviours of the child and its family, to improve diet, physical activity, and the quality of life. Primary care providers play an essential role in motivating, educating, and supporting behavioural modification and maintenance [11].

\section{Disclosure}

The authors declared no conflicts of interest. 


\section{References}

1 Must A, Strauss RS: Risks and consequences of childhood and adolescent obesity. Int J Obes Relat Metab Disord 1999;23(suppl 2):S2-11.

-2 Baker JL, Olsen LW, Sørensen TIA: Childhood body-mass index and the risk of coronary heart disease in adulthood. N Engl J Med 2007;357:2329 2337.

3 Speiser PW, Rudolf MC, Anhalt H, CamachoHubner C, Chiarelli F, Eliakim A, Freemark M, Gruters A, Hershkovitz E, Iughetti L, Krude H, Latzer Y, Lustig RH, Pescovitz OH, PinhasHamiel O, Rogol AD, Shalitin S, Sultan C, Stein D Vardi P, Werther GA, Zadik Z, Zuckerman-Levin N, Hochberg Z: Childhood obesity. J Clin Endocrinol Metab 2005;90:1871-1887.
4 August GP, Caprio S, Fennoy I, Freemark M, Kaufman FR, Lustig RH, Silverstein JH, Speiser PW, Styne DM, Montori VM: Prevention and treatment of pediatric obesity: an endocrine society clinical practice guideline based on expert opinion. J Clin Endocrinol Metab 2008;93:4576-4599.

5 Barlow SE: Expert committee recommendations regarding the prevention, assessment, and treatment of child and adolescent overweight and obesity: Summary report. Pediatrics 2007;120(suppl 4): S164-192.

6 Davis MM, Gance-Cleveland B, Hassink S, Johnson R, Paradis G, Resnicow K: Recommendations for prevention of childhood obesity. Pediatrics 2007; 120(suppl 4):S229-253.
Fernandez JR, Redden DT, Pietrobelli A, Allison DB: Waist circumference percentiles in nationally representative samples of African-American, European-American, and Mexican-American children and adolescents. J Pediatr 2004;145:439-444.

8 Lohman T, Roche A, Martorell R (eds): Anthropometric Standardization Reference Manual. Champaign, IL, Human Kinetics Books, 1988.

9 Kaelber DC, Pickett F: Simple table to identify children and adolescents needing further evaluation of blood pressure. Pediatrics 2009;123:e972-974.

10 Weiss R, Kaufman FR: Metabolic complications of childhood obesity: identifying and mitigating the risk. Diabetes Care 2008;31(suppl 2):S310-316.

11 Flodmark CE, Lissau I, Moreno LA, Pietrobelli A, Widhalm K: New insights into the field of children and adolescents' obesity: the European perspective. Int J Obes Relat Metab Disord 2004;28:1189-1196. 